\title{
INVESTIGATION ON SILICON SOLAR CELL CAPACITANCE AND ITS DEPENDENCE ON BOTH TEMPERATURE AND INCIDENCE ANGLE
}

\author{
Moustapha Sané', Fabé Idrissa Barro \\ 1 Semiconductors and Solar Energy Laboratory, Faculty of Science and Technique, Cheikh Anta Diop University, \\ BP5005, Dakar, Senegal, e-mail: moustaphasane2003@yahoo.fr
}

Received: 2014.09.28

Accepted: 2014.10.25

Published: 2014.12.01

\begin{abstract}
The aim of this work is to investigate a theoretical study of a vertical junction silicon solar cell capacitance under monochromatic illumination. By solving the continuity equation and using a one dimensional model in frequency modulation, we derive the analytical expressions of both excess minority carrier density and photovoltage. Based on these expressions, the solar cell capacitance was calculated; we then exhibited the effects of both temperature and incidence angle on the solar cell capacitance.
\end{abstract}

Keywords: vertical junction, capacitance, temperature, incidence angle.

\section{INTRODUCTION}

It is true that the sun is the principal source of photovoltaic solar energy, but under certain temperatures, it can appear dangerous for the solar cells. Indeed we show how the capacitance compared to the temperature evolves according to certain angles of incidence. In this work, we first derive the excess minority carrier's density and the photovoltage across the junction; these expressions then lead to the solar cell capacitance. The aim of this study is to show the effects of the illumination temperature and incidence angle on the solar cell's capacitance; for that purpose, this capacitance is studied for various incidence angles in frequency modulation.

\section{THEORY}

This study is based on a vertical junction silicon solar cell presented on Figure 1 with the following assumptions:

- The space charge region and the emitter thicknesses are very small compared to that of the base; their contributions are then neglected so that only the contribution of the base is taken into account [1].
- The emitter and the base regions are quasineutrals [2]; there is no internal electric field without the space charge region.

- The electric and optical properties are identical at any point in the base; this enables us to use the Cartesian coordinates. We also took into account the reflection on the silicon material.

- The temperature effect on the performance of the photovoltaic cell is not taken into account.

- The excitation is done with a monochromatic frequency modulated light with wavelength $\lambda$.
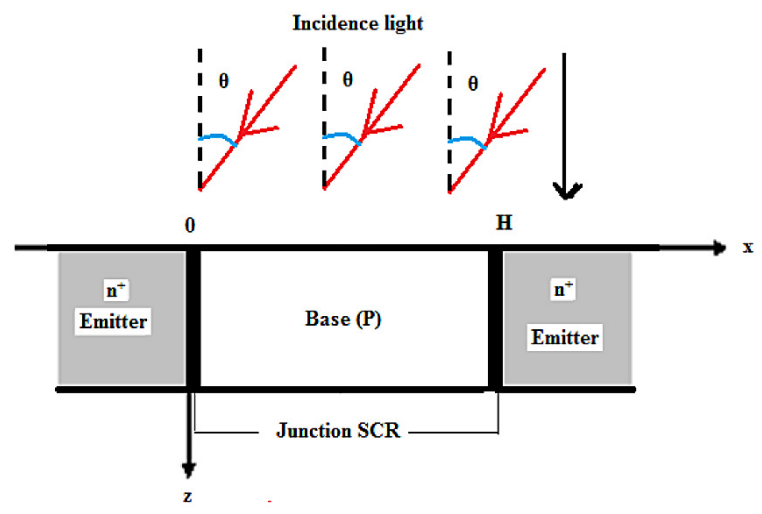

Fig. 1. Vertical junction silicon solar cell with incidence angle 
The incident photons flux $\varphi(\lambda)$ is taken for AM 1.5 solar spectrum $[3,4]$. This case is characteristic of the whole terrestrial applications independently of the processing site. Indeed, the solar radiation corresponds roughly to that of a black body at $6000 \mathrm{~K}$; while crossing the various layers of the atmosphere, certain wavelengths are attenuated by components of the atmosphere such as ozone (absorption of UV - rays in the neighborhoods of $400 \mathrm{~nm}$ ) or the water vapor (absorption of the infrared wavelengths around $800-900 \mathrm{~nm}$ ).

Taking into account the generation, recombination and diffusion phenomena in the base, the equation governing the variation of the minority carrier density $\delta(x, \theta, z, t)$ under modulation frequency $[5,6]$ is:

$D(\omega) \cdot \frac{\partial^{2} \delta(x, \theta, z, t)}{\partial x^{2}}-\frac{\delta(x, \theta, z, t)}{\tau}=-G(z, \theta, t)+\frac{\partial \delta(x, \theta, z, t)}{\partial t}$

where: $D(\omega)$ and $\tau$-are respectively, the excess minority carrier diffusion constant and lifetime,

$\omega$ - the angular frequency,

$x$ - the base depth along $x$ axis,

$\theta$ - the incidence angle, $z$ the base depth according to the vertical axis,

$\delta(x, \theta, z, t)-$ the excess minority carrier density,

$G(\theta, z, t)$ - the carrier generation rate.

The excess minority carrier density can be written as:

$$
\delta(x, t)=\delta(x) \exp (-j \omega t)
$$

The carrier generation rate is given by $[7,8]$ :

$$
G(z, \theta, \lambda, t)=g(z, \theta, \lambda) \exp (-j \omega t)
$$

where the spatial part is given by:

$$
\begin{aligned}
& g(z, \theta, \lambda)=\alpha(\lambda)(1-R(\lambda)) \cdot \phi(\lambda) . \\
& \exp (-\alpha(\lambda) \cdot z) \cdot \cos (\theta)
\end{aligned}
$$

where: $\lambda$ - the illumination wavelength, $\alpha(\lambda)$ and $R(\lambda)$ - are respectively the absorption and reflection coefficients, $t$ - the time and $j^{2}=-1$.

If we replace equation (2) into equation (1), the temporary part $\exp (-j \omega t)$ is eliminated and we obtain:

$$
\frac{\partial^{2} \delta(x)}{\partial x^{2}}-\frac{\delta(x, \theta, t)}{L(\omega)^{2}}=-\frac{g(z, \theta)}{D(\omega)}
$$

where: $L(\omega)$ - is the minority carrier diffusion length.
The solution of this equation (5) is:

$$
\begin{aligned}
& \delta(x, \omega, \theta, z, \lambda)=A \cosh \left(\frac{x}{L(\omega)}\right)+B \sinh \left(\frac{x}{L(\omega)}\right)+ \\
& \frac{L(\omega)^{2}}{D(\omega)} \cdot \alpha(\lambda)(1-R(\lambda)) \cdot \phi(\lambda) \cdot \exp (\alpha(\lambda) \cdot z) \cdot \cos (\theta)
\end{aligned}
$$

Coefficients $A$ and $B$ are determined through the following boundary conditions [4]:

- at the junction $(x=0)$ :

$$
\left.D(\omega) \cdot \frac{\partial \delta(x, \omega, \theta, z, \lambda)}{\partial x}\right|_{x=0}=\left.S f \cdot \delta(x, \omega, \theta, z, \lambda)\right|_{x=0}
$$

where: $S f$ - the junction recombination velocity traducing carrier flow through the junction.

- at the middle of the base $(x=H / 2)$ :

$$
\left.D(\omega) \cdot \frac{\partial \delta(x, \omega, \theta, z, \lambda)}{\partial x}\right|_{x=\frac{H}{2}}=0
$$

where: $H$ - the base depth.

Based on the excess minority carrier density, we can determine the photovoltage across the junction. According to the Boltzmann's relation, the photovoltage is obtained by the expression [9]:

$$
\begin{aligned}
& V(\omega, \theta, z, \lambda, T)=\frac{k \cdot T}{q} . \\
& \ln \left[1+\frac{N b}{n_{0}^{2}} \cdot \delta(0, \omega, \theta, z, \lambda)\right]
\end{aligned}
$$

where: $V_{T}$-the thermal voltage,

$\mathrm{Nb}$ - the base doping density,

$n_{0}$ - the intrinsic carrier density,

$q$ - the elementary charge.

Based on minority carrier density and photovoltage, we can determinate the capacitance of the solar cell. This capacitance is mainly due to the fixed ionized charge (dark capacitance) at the junction boundaries and the diffusion process (diffusion capacitance) [9-12]. The solar cell's capacitance can be defined by:

with:

$$
C(\omega, \theta, z, \lambda, T)=\frac{d Q(\omega, \theta, z, \lambda)}{d V(\omega, \theta, z, \lambda, T)}
$$

$$
Q(\omega, \theta, z, \lambda)=\left.q \delta(x, \omega, \theta, z, \lambda)\right|_{x=0}
$$

Given the photovoltage expression, eq. 10 can be rewritten as:

$$
\begin{aligned}
& C(\omega, \theta, z, \lambda, T)= \\
& \frac{q^{2}}{k \cdot T} \cdot\left(\frac{n_{i}^{2}}{N b}+\delta(0, \omega, \theta, z, \lambda)\right)
\end{aligned}
$$


Under illumination, the diffusion capacitance predominates. Based on all the previous expressions and some computation, we obtained the behavior of the solar cell capacitance presented on the next section.

\section{RESULTS AND DISCUSSION}

Based on the mathematical formulation, simulation where performed from various induced fields through different forward biased voltages and various external load conditions through the dynamic junction velocity.

We present the capacitance of the solar cell versus temperature for various incidence angles (Figure 2).

Figure $2 \mathrm{a}$ shows that the capacitance of solar cell decreases in an exponential way when the temperature increases within the interval $(100 \mathrm{~K}$; $380 \mathrm{~K})$. Indeed, the increase in the temperature causes a certain flexibility of the solar cell allowing the minority of carriers diffuse more easily. This allows the base and the junction to empty their carriers which will take part in the photocurrent. We can also observe a decrease of the capacitance for the chosen incidence angles; effectively, when incidence angles increases, the incident photons flux penetrating the solar cell decreases due to reflexion coefficient; this lead to a decrease of excess carrier's concentration in the base and to an increase of the base dynamic mobility and thus its resistivity; that why the capacitance decrease for a given operating point.

Figure $2 \mathrm{~b}$ gives the profiles of capacitance versus temperature reverse and show that the slope of the straight line is $1 / \mathrm{Vt}$ and it intercept the temperature axis at a value corresponding to the activation temperature $1 /$ To. We also see that the more the incidence angle increases the more the activation temperature decreases. Effectively, the increase of incidence angle traduces the decrease of the incident photons flux so the decrease of the sun intensity.

The figure $2 \mathrm{c}$ gives the profiles of capacitance reverse versus temperature and shows that the slope of the straight line is $1 / \mathrm{Vt}$ and it intercept the capacitance axis at a value corresponding to the dark capacitance $1 / \mathrm{Co}$. We also note that the value of the dark capacitance decreases when the incidence angle increases. Indeed, this decrease is due to the fall of sun intensity, thus generating fewer carriers.
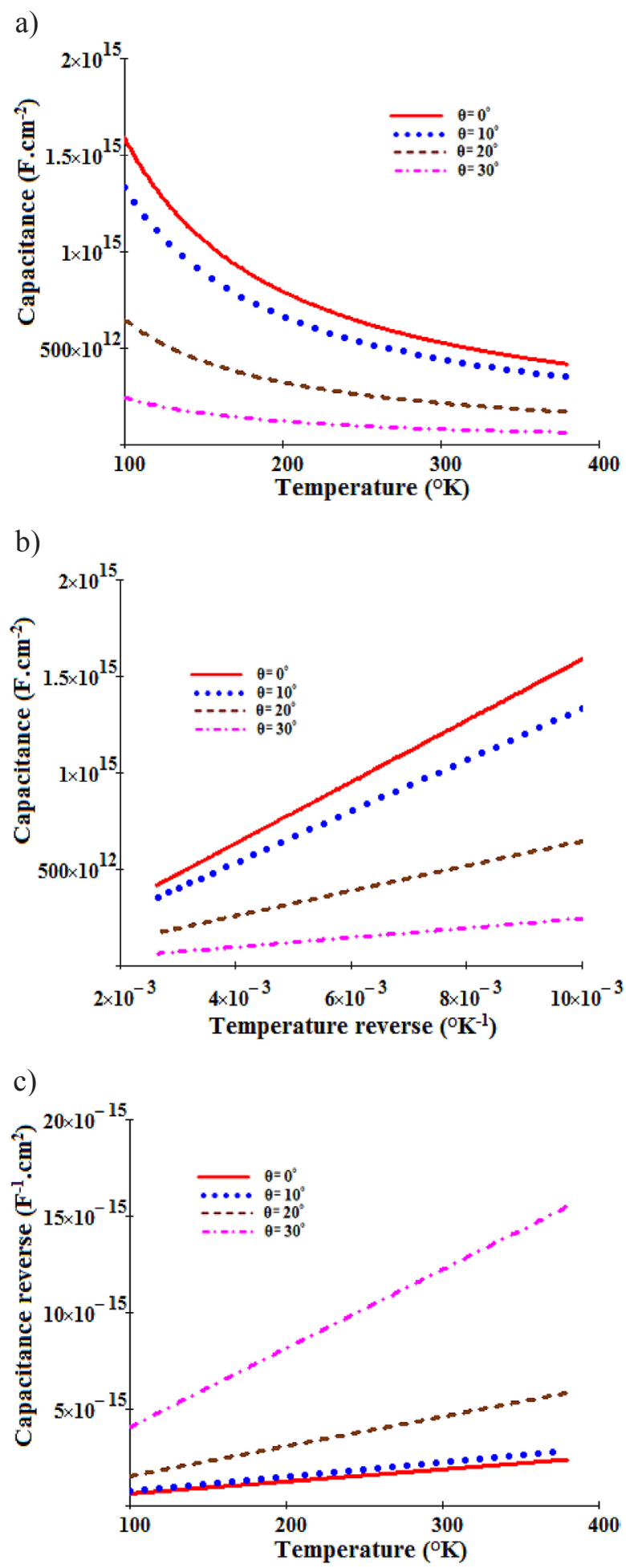

Fig. 2. Capacitance of silicon solar cell behavior under temperature for various incidence angle

\section{CONCLUSION}

A theoretical study of a vertical junction of solar cell has been presented. Based on the excess minority carriers' density and the photovoltage, the solar cell capacitance has been calculated. 
This capacitance is then studied for various incidence angle and we showed that solar cell's capacitance strongly depends on both incidence angle. For the considered incidence angles range, the capacitance decreases with increasing incidence angle.

\section{REFERENCES}

1. Sze S.M., Kwok K.N.: Physics of semiconductors devices. Wiley, New York, NY, USA, 2007.

2. Sané M., Zoungrana M., Diallo H.L., Sahin G., Thiam N., Ndiaye M., Dieng M., Sissoko G.: Influence of incidence angle on the electrical parameters of a vertical silicon solar cell under frequency modulation. International Journal of Inventive Engineering and Sciences, Vol. 1(11), 2013, 37-40.

3. Sané M., Sahin G., Barro F.I., Maiga A.S.: Incidence angle and spectral effects on a vertical junction silicon solar cell capacitance. Turkish Journal of Physics, Vol. 38, 2014, 221-227.

4. Barro F.I., Sané M., Sahin G.: Silicon solar cell space charge region and capacitance behavior under electric field. Sylwan, Vol. 158(5), 58-66.
5. Sané M., Zouma B., Barro F.I.: New approach to determine vertical parallel junction silicon solar cell parameters. Research Journal of Applied Sciences, Engineering and Technology, Vol. 7(24), 2014, 5288-5292.

6. Mathieu H., Fanet H.: Physique des semiconducteurs et des composants électroniques. $6^{\text {th }}$ Ed., Dunod, 2009.

7. Green M.A., Emery K., Hishikawa Y., Warta W.: Progress in photovoltaics. Research and applications, Vol. 18, 2010, 144-150.

8. Green M.A.: Solar energy. Material and Solar Cells. Vol. 92, 2008, 1305-1310.

9. Sané M.: Study of vertical junction polycristalline silicon solar cell in frequency modulation under monochromatic illumination with incidence angle. Doctorate Thesis, Department of Physics, Cheikh Anta Diop University, Dakar, Senegal, 2014.

10. Hu C.C.: Modern semiconductor devices for integrated circuits. Pearson/prentice Hall, New Jersey, 2010.

11. Boer K.W.: Introduction to space charge effects in semiconductor. Springer-Verlag, 2010.

12. Barsoukov E., Macdonald J.R., Impedance Spectroscopy: Theory, Experiment and Applications. Wiley: New York, 2005. 